\title{
Further resolution of the house mouse (Mus musculus) phylogeny by integration over isolation-with-migration histories
}

\author{
Megan Phifer-Rixey ${ }^{1 *} \mathbb{D}$, Bettina Harr ${ }^{2}$ and Jody Hey ${ }^{3}$
}

\begin{abstract}
Background: The three main subspecies of house mice, Mus musculus castaneus, Mus musculus domesticus, and Mus musculus musculus, are estimated to have diverged $\sim 350-500 \mathrm{KYA}$. Resolution of the details of their evolutionary history is complicated by their relatively recent divergence, ongoing gene flow among the subspecies, and complex demographic histories. Previous studies have been limited to some extent by the number of loci surveyed and/or by the scope of the method used. Here, we apply a method (IMa3) that provides an estimate of a population phylogeny while allowing for complex histories of gene exchange.

Results: Results strongly support a topology with M. m. domesticus as sister to M. m. castaneus and M. m. musculus. In addition, we find evidence of gene flow between all pairs of subspecies, but that gene flow is most restricted from M. m. musculus into M. m. domesticus. Estimates of other key parameters are dependent on assumptions regarding generation time and mutation rate in house mice. Nevertheless, our results support previous findings that the effective population size, $N_{e}$, of M. m. castaneus is larger than that of the other two subspecies, that the three subspecies began diverging $\sim 130-420 \mathrm{KYA}$, and that the time between divergence events was short.
\end{abstract}

Conclusions: Joint demographic and phylogenetic analyses of genomic data provide a clearer picture of the history of divergence in house mice.

Keywords: Speciation, Divergence, Population size, IMa3

\section{Background}

The house mouse (Mus musculus) has long been a genetic model for human biology and disease (reviewed in [1-3]). House mice have also grown into a model system for evolutionary genetics, fueling investigations of topics ranging from meiotic drive to adaptive introgression (e.g. $[4,5])$. In particular, studies in house mice have shed light on the process of speciation and the genetic basis of reproductive isolation (e.g. [6-14]). More recently, studies have leveraged the increasing geographical distribution of house mice to investigate the genetics

\footnotetext{
* Correspondence: mphiferr@monmouth.edu

'Department of Biology, Monmouth University, West Long Branch, NJ, USA Full list of author information is available at the end of the article
}

of phenotypic change and adaptation accompanying range expansion (e.g. $[15,16])$. However, much of this work relies on an understanding of the evolutionary history of house mice that continues to be refined.

The most numerous subspecies of house mice, Mus musculus domesticus, Mus musculus musculus, and Mus musculus castaneus, are found over different, but overlapping, geographical ranges (reviewed in [17]). While the subspecies can be crossed in the lab, in some cases, hybrid males are sterile or have reduced fertility (e.g., [7, $18,19])$. There is extensive evidence of hybridization between the subspecies in the wild. The hybrid zone between $M . m$. domesticus and M. m. musculus has been particularly well-studied (for review, see [20]), but M. $m$. domesticus in the United States harbor introgression

(c) The Author(s). 2020 Open Access This article is licensed under a Creative Commons Attribution 4.0 International License, which permits use, sharing, adaptation, distribution and reproduction in any medium or format, as long as you give appropriate credit to the original author(s) and the source, provide a link to the Creative Commons licence, and indicate if changes were made. The images or other third party material in this article are included in the article's Creative Commons licence, unless indicated otherwise in a credit line to the material. If material is not included in the article's Creative Commons licence and your intended use is not permitted by statutory regulation or exceeds the permitted use, you will need to obtain permission directly from the copyright holder. To view a copy of this licence, visit http://creativecommons.org/licenses/by/4.0/ The Creative Commons Public Domain Dedication waiver (http://creativecommons.org/publicdomain/zero/1.0/) applies to the data made available in this article, unless otherwise stated in a credit line to the data. 
from $M . m$. castaneus [21] and there is evidence of a hybrid zone in China between M. m. castaneus and M. $m$. musculus [22]. In fact, evidence suggests that another subspecies in the group, Mus musculus molossinus, found in Japan, was formed by hybridization between $M$. m. castaneus and M. m. musculus [23].

The group is believed to have originated in Southwestern Asia [24, 25] and analyses support a near simultaneous divergence between the three subspecies within the last $\sim 350,000-500,000$ years (e.g., [25-28]). The demographic histories of the subspecies differ markedly. The center of diversity for M. m. castaneus is also in Southwestern Asia and estimates of effective population size $\left(N_{e}\right)$ of $M$. m. castaneus are large ( 200,000-700,000 [27-29]). On the other hand, estimates of $N_{e}$ for $M$. m. domesticus ( 58,000-200, 000) and M. m. musculus (25,000-120,000 [27, 28, 30]) are much smaller. Both are believed to have undergone bottlenecks as they shifted their rangesM. $m$. domesticus through the Middle East and North Africa into Western Europe and M. m. musculus into North Asia and Eastern Europe [25, 26].

The combination of a relatively recent divergence, ongoing gene flow, and complex demography complicates the resolution of the phylogenetic relationships between the subspecies. Nevertheless, previous studies have provided fundamental insights into the history of their divergence. Geraldes et al. [27] and Geraldes et al. [28] sampled many individuals of all three subspecies $(n=$ 26-60) across a modest number of loci (8 and 27 respectively) and used multiple two-population isolation-with-migration (IM) models [31-33] to estimate key parameters, e.g., $N_{e}$, migration rates, and divergence times. However, resolution of the topology of the subspecies group using a pairwise approach was not possible and resolution of divergence times was limited. In some instances, it was not possible to obtain either a reliable estimate of divergence time or confidence intervals for estimates of divergence time. In other analyses, confidence intervals were large [27, 28]. Suzuki et al. [25] found more topological resolution by applying phylogenetic methods to mtDNA from a sample of mice spanning Europe, Asia, Africa, and Australia. Their results support M. m. domesticus as sister to M. $m$. castaneus and M. $m$. musculus, and provide evidence for a split between $M$. $m$. domesticus and (M. m. castaneus, M. m. musculus) 459KYA (CI: 325 - 481KYA). However, reliance on mtDNA alone can be problematic when estimating phylogenies (e.g., [34]). A different approach, Bayesian concordance analysis, uses genome wide data from single individuals of each subspecies to estimate gene trees. Two such studies have considered the topology of the house mouse subspecies [35, 36]. Both find support for M. m. castaneus and M. m. musculus as sister to M. $m$. domesticus, but the alternate topologies have moderate support, highlighting extensive phylogenetic discordance in the group. For example, in White et al. [36] only 39\% of gene trees supported the primary topology compared to the $33 \%$ expected under a simultaneous divergence. In addition, this approach is not useful for estimating other parameters of interest.

Here, we revisit the history of divergence among the subspecies by taking advantage of a new method that allows for phylogeny estimation with multiple species or populations that is especially useful in this potential trichotomy (IMa3 [37];). IMa3 is a genealogy sampling program [38] that implements a multi-population IM model with a novel "hidden genealogy" Markov-chain Monte Carlo (MCMC) update that permits the sampling of population phylogenies [37]. Once an estimate of the posterior probability distribution of population phylogenies has been obtained, the program can be run a second time, while fixing the phylogeny on the estimated value, in order to obtain estimates for $N_{e}$ values, migration rates and branch lengths (splitting times). With these two successive runs, the method provides for a joint estimate of the rooted population phylogeny and the complex demographic history within that phylogeny [37]. We applied this approach to publicly available genomic data, incorporating 200 randomly selected autosomal loci for multiple individuals of each subspecies ([39]; Table S1). Our results strengthen and refine our understanding of divergence among house mouse subspecies. Most notably, we found strong support for a sister relationship between $M . m$. musculus and M. m. castaneus and that divergence among the three species likely began no more than $\sim 500 \mathrm{KYA}$ and possibly as recently as 130KYA.

\section{Results and discussion}

IMa3 analysis of 200 randomly selected autosomal loci supported a phylogeny with $M . m$. musculus and $M . m$. castaneus as sister to $M$. m. domesticus, with or without the inclusion of an unsampled 'ghost' population (Fig. 1, Table 1). Without a ghost population, the posterior probability for that topology was 0.759 compared to 0.146 for a sister relationship between $M$. m. castaneus and $M$. m. domesticus, and 0.096 for a sister relationship between $M . m$. musculus and $M . m$. domesticus. With a ghost population, the posterior probability for that topology was 0.921 compared to 0.050 for a sister relationship between $M . m$. castaneus and M. m. domesticus, and 0.029 for a sister relationship between $M . m$. domesticus and M. m. musculus. Overall, there is little evidence that an unsampled population has shaped the demographic history of these three subspecies, given that the estimates of the posterior distribution of phylogenies, as well as parameter estimates (Table 1; Tables S2, S3, 


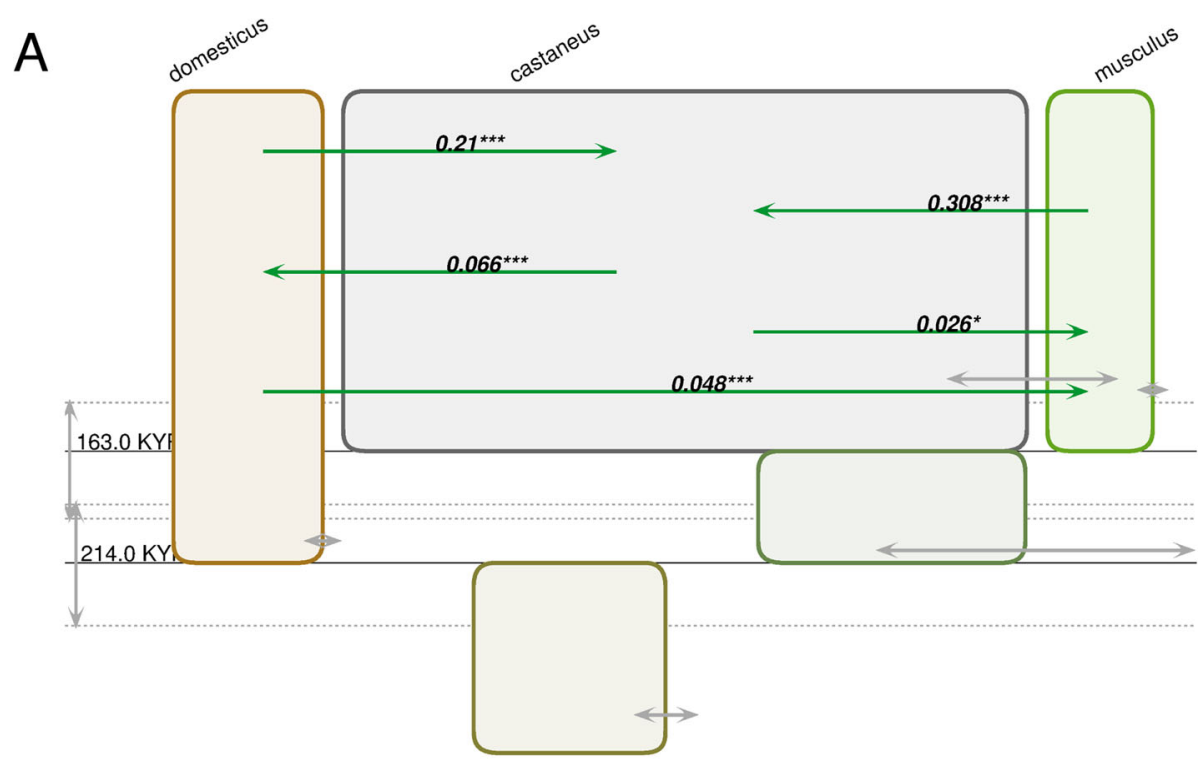

Ancestral $\mathrm{Ne}$ (thousands): 140.0

B

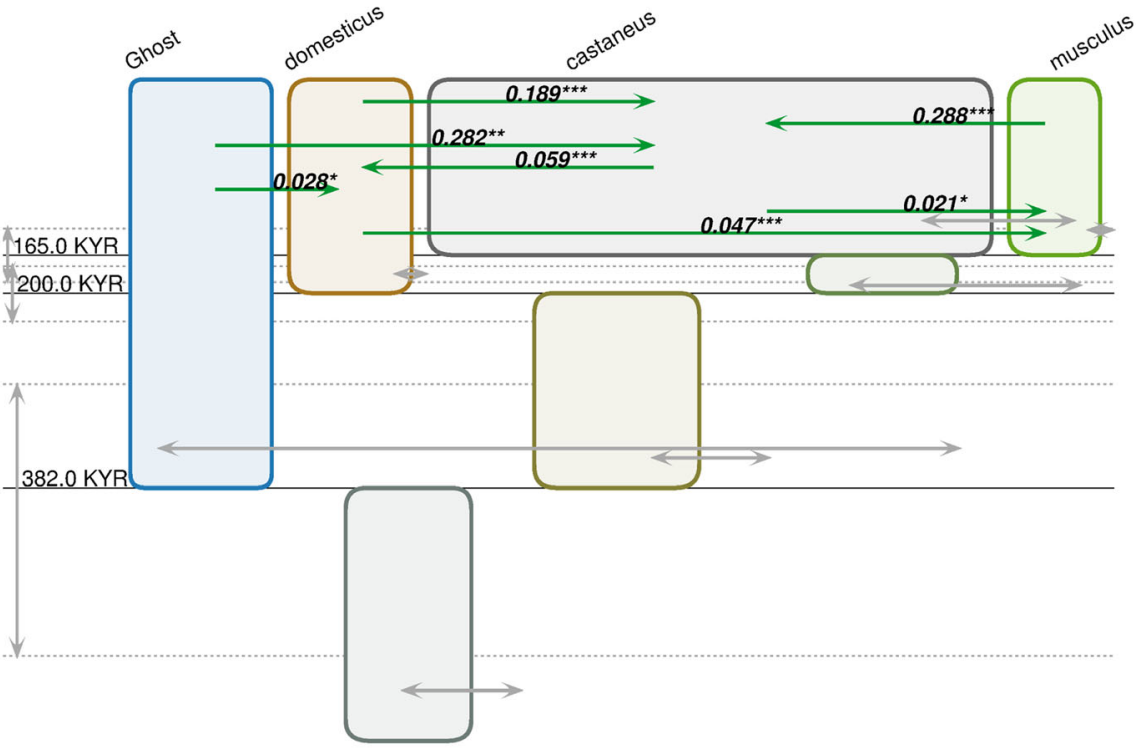

Ancestral Ne (thousands): 98.9

Fig. 1 Representations of estimated IM models generated by IMa3 and the IMfig program [37] for the three subspecies of house mouse. The phylogeny is depicted as a series of boxes organized hierarchically, with ancestor boxes positioned in between the corresponding descendants, and the width of boxes proportional to estimated $N_{e}$. Gray arrows extending to the left and right of the right boundary of each population box depict $95 \%$ confidence intervals for $N_{e}$ values. Splitting times are depicted as solid horizontal lines, with text values on the left. Confidence intervals for splitting times are shown as vertical gray arrows on the left, and parallel dashed lines. Migration arrows (if shown) indicate estimated $2 \mathrm{Nm}$ values from one population to another over the time interval when both populations exist. Arrows are shown only for estimated migration rates that are statistically significant at or above the 0.05 level $\left({ }^{*} p<0.05,{ }^{* *} p<0.01,{ }^{* * *} p<0.001\right.$ [33]). (a) without a ghost population (b) with a ghost population included. Estimates assume 1.5 generations/year and $6 \times 10^{-9}$ mutations per base pair per generation

S4, S5, S6 and S7), are similar in runs that did and did not include a ghost population.

To convert parameter estimates, which are scaled by mutation rate [31], to useful demographic scales, a mutation rate and generation time are required. We assumed 1.5 generations per year, which is intermediate between reported frequencies of one and two generations per year $[27,40]$. For mutation rate, we used a recent estimate based on trio sequencing of $6 \times 10^{-9}$ mutations per base pair per generation [41]. This is 
Table 1 The topology that places M. m. castaneus sister to M. m. musculus was sampled most frequently, whether a ghost population was included or not. The three subspecies are numbered from 0 to 2 (corresponding to M. m. castaneus, M. $m$. domesticus, and M. m. musculus, respectively). Ancestral populations are numbered beginning with 3 and are ordered numerically in time (i.e. 4 is the ancestor of all populations). When a ghost population is included, it is an outgroup to the sampled subspecies and has not been included in the species tree notation

\begin{tabular}{llll}
\hline Model & Species Tree & Count & Frequency \\
\hline Without ghost & $(1,(0,2) 3) 4$ & 237,697 & 0.758636 \\
& $(2,(0,1) 3) 4$ & 45,610 & 0.145753 \\
With ghost & $(0,(1,2) 3) 4$ & 29,919 & 0.095610 \\
& $(1,(0,2) 3) 4$ & 227,602 & 0.920739 \\
& $(2,(0,1) 3) 4$ & 12,363 & 0.050013 \\
& $(0,(1,2) 3) 4$ & 7230 & 0.029248 \\
\hline
\end{tabular}

similar to a previous phylogeny-based estimate of $4.2 \times$ $10^{-9}$ [42] and to an autosomal estimate from [27] $(4.1 \times$ $\left.10^{-9}\right)$. For clarity, unless otherwise noted, we report scaled estimates based on these assumptions. However, for more direct comparison to previous studies, we also provide estimates scaled using 1 and 2 generations/year and $4.1 \times 10^{-9}$ mutations per base pair per generation (Tables S2, S3, S4, S5 and S6).

Estimates of $N_{e}$ for each of the subspecies were consistent with previous work (Tables S5, S6 [27-30]). Given our assumptions for mutation rate and generation time, $M . m$. castaneus had the largest estimated $N_{e}$, with confidence intervals from both models (with and without a ghost population) ranging from $\sim 419,000-569,000$. M. m. domesticus was intermediate, with estimates ranging from $\sim 91,000-121,000$ and the smallest estimates of $N_{e}$ were from M. m. musculus, $\sim 67,000-86,000$ (Table S5-S6). As with previous studies, estimates of ancestral $N_{e}$ were of the same order of magnitude as estimates for the extant subspecies (without ghost: 139,951 CI: 118,420-163,709; with ghost: 105,347 CI: 41,320-147, 783; Table S5, S6 [27, 28]).

For all migration rate parameters, in both directions between pairs of subspecies, the $95 \%$ confidence interval for estimates of migration did not include zero. In addition, estimates of migration rate were statistically significant, whether including a ghost population or not, with the exception of migration from $M . m$. musculus into M. m. domesticus (Fig. 1; Tables S2, S3, S7). In some cases, such as gene flow into $M . m$. castaneus from the other two subspecies, the estimated population migration rate $(2 \mathrm{Nm}$ ) was quite high (e.g., 0.210 and 0.308 from $M . m$. domesticus and $M$. m. musculus, respectively, in the analysis without a ghost population, Table S7). While estimates of migration rates were generally reported as not statistically significant in previous studies, patterns of gene flow inferred here are broadly consistent with previous findings that reject a model of speciation with no gene flow and suggest more migration into $M . m$. castaneus than into either of the other two subspecies $[27,28]$. A model of speciation with reciprocal migration, but that is more limited into $M . m$. domesticus and M. m. musculus, is also consistent with results from studies of contemporary hybrid zones, laboratory crosses, and genome-wide patterns of genetic variation. Hybridization occurs between each pair of subspecies in the wild (e.g., [22, 24, 26, 43]), but the degree of reproductive isolation observed in the lab differs. Crosses between M. m. domesticus and M. m. musculus can result in significant male sterility (e.g., [13, 44]), while impacts on male fertility are not observed until the $\mathrm{F}_{2}$ in crosses between $M . m$. castaneus and $M . m$. domesticus, [19] and have not been reported at all in crosses between $M . m$. castaneus and $M . m$. musculus. Levels of genetic differentiation between $M . m$. musculus and $M$. $m$. domesticus are also higher than between any other pair of subspecies (e.g., [11, 28]).

The estimated history given the dominant phylogeny and our assumptions regarding mutation rate and generation time suggests all three subspecies diverged within the last 250,000 years (Fig. 1; Tables S2, S3 and S4). Divergence between $M . m$. domesticus and (M. m. castaneus, M. m. musculus) was estimated at 214,158 KYA (CI: 188,767-243,559) and the subsequent divergence between $M . m$. castaneus, $M . m$. musculus was estimated at 163,375 KYA (CI: 142,661-194,781). Including a ghost population shifted these estimates slightly to 200,010 KYA (CI: 175,888-227,482) and 165,167 KYA (CI: 141, 716-189,959), respectively. Our main analysis was limited to runs with a maximum of 200 intergenic loci because of time and MCMC mixing constraints. One benefit of increasing genomic and computational resources is that we can assess the effect of sampling on our results using a separate analysis with 200 different loci, sampled at random using the same protocol as the primary set. The phylogeny estimates and the IM model estimates with this second set of 200 randomly selected loci were very similar to the primary analysis (Table S8, S9, S10 and 11 and Figure S1).

While these estimates are more recent than reported in most previous studies, it is important to note that estimates of divergence time in years are sensitive to assumptions regarding generation time and mutation rates. Assuming a generation time of 1 year and a mutation rate of $4.1 \times 10^{-9}$, Geraldes et al. [27] estimated the divergence time of $M . m$. domesticus and $M . m$. castaneus as $~ 330$ KYA (90\% posterior density interval: 220, 897 -579,617). For M. m. domesticus and M. m. musculus, divergence time was estimated as $\sim 628 \mathrm{KYA}$ (no CI 
reported). Divergence time between $M . m$. castaneus and $M . m$. musculus could not be reliably estimated. Given that ML estimates were unreliable and confidence intervals were large, the authors concluded that divergence occurred in the last $~ 500 \mathrm{KYA}$. Assuming a generation time of 1 year, results from Geraldes et al. [28] suggested a near simultaneous divergence among the three subspecies $\sim 350 \mathrm{KYA}$ (M. m. castaneus and M. $m$. musculus: 320,764 KYA, no CI reported; M. m. domesticus and M. m. musculus: 345,752 KYA, no CI reported; M. m. domesticus and M. m. castaneus: 313,822 KYA, CI: 247,268-372,981). Rescaling our results following assumptions in Geraldes et al. [27] yields broadly consistent divergence time estimates of $\sim 400 \mathrm{KYA}$ for $M . \mathrm{m}$. domesticus and (M. m. castaneus, M. m. musculus) and $\sim 320$ KYA for the subsequent split between M. m. castaneus, M. m. musculus (Table S4).

The general agreement among the studies is notable, especially given differences in the number and nature of loci sampled and the geographic range of sampling. Geraldes et al. [27] included just eight loci, including three that were sex-linked and one that was mitochondrial, and sampled intronic regions rather than the intergenic regions sampled in our study. Geraldes et al. [28] surveyed mostly intronic regions of 27 autosomal loci. In addition, while the geographic regions sampled in our study are very similar to those sampled in Geraldes et al. [28], they are a subset of those included in Geraldes et al. [27]. In particular, Geraldes et al. [27] sampled $M$. $m$. castaneus from China and Taiwan in addition to India. If the additional populations included in Geraldes et al. [27] were more divergent, this would be expected to drive up polymorphism levels and $N_{e}$ estimates within subspecies. We did not observe this, and our estimates for $N_{e}$ in $M . m$. castaneus are as high or higher than reported by Geraldes et al. [27]. Moreover, comparisons of population genetic summary statistics suggest that levels of nucleotide variation among the autosomal loci included in the Geraldes et al. [27] study and our random sample of 200 loci are similar (Tables S12, S13 and S14 [45];).

We also reanalyzed data from Geraldes et al. [27] to more directly compare our results to those of previous isolation-with-migration analyses on pairs of species. With a pairwise approach, there was no resolution of the topology. However, our reanalysis including four autosomal and two X-linked loci from [27] supported the (Mus musculus domesticus, (Mus musculus castaneus, Mus musculus musculus)) phylogeny (estimated posterior probability 0.479 ) compared to the other possible topologies (estimated posterior probabilities 0.315 and 0.206, Table S15). Therefore, while there were differences in some estimates of demographic parameters (e.g. ancestral $N_{e}$ and divergence times), the overall topology was recovered using IMa3 even with this limited set of loci (Fig. 2, Tables S15, S16, S17 and S18).

\section{Conclusions}

Our joint demographic and phylogenetic analyses refine our understanding of the history of divergence in house mice. Results significantly strengthen evidence for a sister relationship between $M . m$. castaneus and $M$. $m$. musculus $([25,35,36])$. The IMa3 analyses also found evidence of gene flow between all pairs of subspecies, but that gene flow into $M . m$. domesticus from $M . m$. musculus was more limited. Estimates of effective population size for the extant subspecies are largely consistent with previous results, with the estimates of $N_{e}$ in $M$. $m$. castaneus of on the order of $\sim 350,000-1,100,000$, estimates for $M$. m. domesticus of $\sim 80,000-240,000$, and estimates for M. m. musculus of $\sim 60,000-170,000$. Estimates of divergence time suggest that $M . m$. domesticus split from (M. m. castaneus and M. m. musculus) in the last 500KYA and potentially as recently as $130 \mathrm{KYA}$ and that the split between $M . m$. castaneus and $M . m$. musculus occurred shortly thereafter, 110-320KYA.

\section{Methods}

\section{Sequence data}

Genomic data were derived from Harr et al. [39]. Briefly, DNA was extracted from samples of mice collected throughout the range of the subspecies in Europe and Asia via either DNeasy kits (Qiagen, Hilden, Germany) or salt extraction [46]. Libraries were then prepared via the TruSeq DNA LT Sample Prep Kit v2 or the Nextera DNA library Prep Kit. Paired-end sequencing of the libraries was performed on either the Hiseq2000 or the NextSeq 500. These reads along with previously published $M . m$. castaneus [29] reads were then mapped to the mm10 genome reference sequence [47] via bwamem [48] and a bioinformatics pipeline including Picard (http://broadinstitute.github.io/picard/) and GATK [49] was used to remove duplicates and call variants. Coverage varied among individuals and there was some evidence for relatedness among individuals within subspecies [39]. In addition, there were many more individuals re-sequenced from $M . m$. domesticus and $M . m$. musculus than M. m. castaneus. To generate the data file for subsequent analyses, we selected a subset of samples from each subspecies, avoiding individuals with high relatedness scores and/or lower coverage and maximizing geographic representation ( $\mathrm{S} 1 ; M . m$. castaneus, $n=7$; M. $m$. domesticus, $n=9 ; M$. m. musculus, $\mathrm{n}=9$ ). Starting from the full population-sorted vcf file (available here, http://wwwuser.gwdg.de/ evolbio/evolgen/wildmouse/vcf), we used GATK to filter out all other individuals and nonvariant sites starting with the file including only SNPs that were flagged "PASS" (for filtering details, see [39]). 


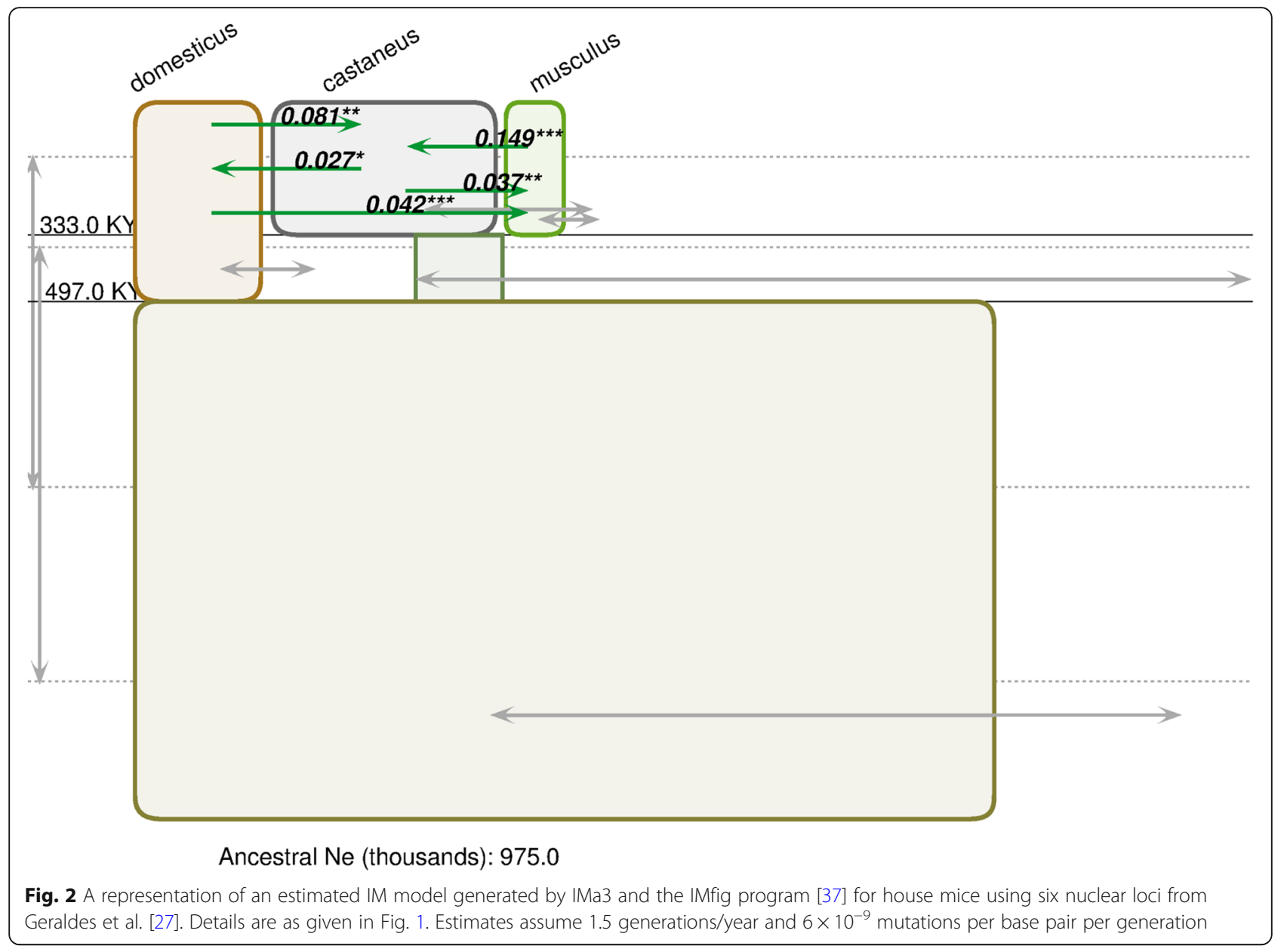

\section{IMa3 analyses}

Like other genealogy samplers, IMa3 is limited by the assumptions that loci are separated by high recombination, whereas recombination within loci is absent. To generate sampled regions that do not show evidence of recombination, regions were subsampled using the 4-gamete criterion [50]. However, this type of filter does not account for the effect of recombination events that are not detected. To minimize these effects, we sampled nonoverlapping regions that passed the 4-gamete criterion, with a minimum number of two SNPs for each sampled region [51] as previously described [52]. Because the genealogies of all the loci must be updated simultaneously in IMa3, when updating the phylogeny [37], runtimes increase greatly when large numbers of loci are used, even when using multiple processors. Given runtime considerations, and from previous experience [37], we focused on data sets of 200 loci. Runs with larger numbers of loci were attempted (e.g. 400 loci), however the Markovchain simulations showed poor mixing. Sampled regions were selected to exclude: [4] regions within 10,000 base pairs of coding regions, because of possible selective effects [20]; CpG sites, because of the possibility that SNPs in these positions could be caused by more than 1 mutation; and [24] simple repeats, because of possible misalignment within and near repeats. Files were prepared using modifications of scripts available from the PopGen Pipeline Platform [53]. For the 200 sampled loci, the mean locus length was 544 base pairs and the mean number of polymorphic sites was 12.9 (Table S19). Linked selection can create covariation between recombination rate and local estimates of $N_{e}$. To address this possible bias, we also calculated the recombination rate at each locus using a sex averaged map [54] and the Mouse Map Converter (http://cgd.jax.org/mousemap converter; Table S19). Recombination rates were calculated by obtaining genetic map positions to either side of the locus $(+/-10,000 \mathrm{bp}$ on either side) and estimating the derivative (rate) by taking the difference in map position and dividing by $(20,000+$ locus length). We found no significant correlation between recombination rate and variability in our dataset (Figure S2; $\mathrm{y}=0.0005 x+$ $0.0272, \mathrm{R}^{2}=0.0009$ ).

IMa3 runs used uniform prior distributions with upper bounds of 2.0 for population size mutation rate $(4 N \mu)$ parameters, 0.2 for migration rates $(\mathrm{m} / \mu)$ and 1.5 for 
splitting time $(t \mu)$. In order to ensure proper mixing of the Markov-chain simulation, a large number (400-480 depending on the run) of Metropolis-coupled [55] chains were used, with 80 or 100 processors for each run. Runs began with a burn-in period of $24 \mathrm{~h}$, followed by 3 or 4 days of sampling. Effective sample sizes were estimated over 1500 phylogenies for all runs. We repeated our analyses following the same protocol with a second set of 200 randomly sampled loci.

For comparison, we also analyzed six loci from Geraldes et al. [27], four on the autosomes and two on the $\mathrm{X}$ chromosome. These data were originally analyzed using the older IM program [31] in three pairwise analyses. We aligned the data (A. Geraldes, pers. comm.) for all three species and sampled intervals and assigned mutation rates as given above for the analysis of the 200 locus autosomal data set. Because these loci were longer on average than those used for our main analysis, the uniform prior distributions for the IMa3 run had upper bounds of 10.0 for population size mutation rate $(4 N \mu)$ parameters, 0.2 for migration rates $(\mathrm{m} / \mu)$, and 1.5 for splitting time $(t \mu)$. A ghost population was not included in the model. A 14-h run (2-h burn-in) using 90 chains and 30 processors yielded results with effective sample sizes above 500 .

\section{Supplementary information}

Supplementary information accompanies this paper at https://doi.org/10. 1186/s12862-020-01666-9.

Additional file 1:Supplementary Table 1. Collection information and mapping metrics for samples from Harr et al. [39] included in this study. Supplementary Table 2. Parameter estimates with 95\% confidence limits for the most probable model of three subspecies without a ghost population. Supplementary Table 3. Parameter estimates with 95\% confidence limits for the most probable model of three subspecies with a ghost population. Supplementary Table 4. Estimated split times (years) with 95\% confidence limits. Supplementary Table 5. Estimated effective population sizes $\left(N_{e}\right)$ with $95 \%$ confidence limits (no ghost population). Supplementary Table 6. Estimated effective population sizes $\left(N_{e}\right)$ with $95 \%$ confidence limits (with a ghost population). Supplementary Table 7. Estimated population migration rates with 95\% confidence limits for the most probable topology with and without a ghost population included. Supplementary Table 8. Phylogeny results from the analysis of a second set of 200 random autosomal loci without a ghost population (compare with values from Table 1). Supplementary Table 9. Parameter estimates with 95\% confidence limits for the most probable model of three subspecies without a ghost population for a second set of 200 random autosomal loci.

Supplementary Table 10. Estimated split times (years) and effective population sizes $\left(\mathrm{N}_{e}\right)$ with $95 \%$ confidence limits using a second set of 200 random autosomal loci (without a ghost population).

Supplementary Table 11. Estimated population migration rates with 95\% confidence limits for the most probable topology using a second set of 200 random autosomal loci (without a ghost population).

Supplementary Table 12. $\theta^{\wedge} \pi$ / $D^{\wedge} x y$ matrix for the 200 randomly selected autosomal loci from the genomic data. Supplementary Table 13. $\theta^{\wedge} \pi / D^{\wedge} x y$ matrix for the 4 autosomal loci from Geraldes et al. [27]. Supplementary Table 14. $F_{s t}$ between subspecies was calculated as the mean across loci. Locus specific values of $F_{\text {st }}$ were calculated using pairwise differences between sequences following Hudson, Slatkin, and
Maddison [45]. Supplementary Table 15. Phylogeny results from the re-analysis of four autosomal and two X-linked loci included in Geraldes et al., [27]. Supplementary Table 16. Parameter estimates with 95\% confidence limits for the most probable model of three subspecies using four autosomal and two X-linked loci included in Geraldes et al. [27]. Supplementary Table 17. Estimated split times (years) and effective population sizes $\left(N_{e}\right)$ with $95 \%$ confidence limits for an analysis using four autosomal and two X-linked loci included in Geraldes et al. [27]. Supplementary Table 18. Estimated population migration rates with $95 \%$ confidence limits given the most probable topology for an analysis using four autosomal and two X-linked loci included in Geraldes et al. [27]. Supplementary Table 19. Summary information for 200 loci, including location on the chromosome, length, number of variable sites, and recombination rate (cM/Mb). Recombination rates were based on sexaveraged maps [54] as reported at http://cgd.jax.org/mousemapconverter/. Supplementary Figure 1. A representation of an estimated Isolation with Migration model generated by IMa3 and the IMfig program [37] for house mice using a set of 200 alternative random autosomal loci. Details are as given in Fig. 1. Estimates assume 0.75 generations/year and $6 \times 10^{-9}$ mutations per base pair per generation. Supplementary Figure 2. SNP density plotted against recombination rate for 200 sampled loci for A) all loci $\left(y=0.0005 x+0.0272, R^{2}=0.0009\right)$ and $\left.\mathbf{B}\right)$ all loci excluding outliers with recombination rate greater than $2 \mathrm{cM} / \mathrm{Mb}(y=0.0008 x+$ $0.271, R^{2}=0.0007$; data from Table S19).

\section{Abbreviations}

bp: Basepair; Cl: Confidence interval; IM: Isolation with migration; MCMC: Markov chain Monte Carlo; $\mathrm{N}_{e}$ : Effective population size

\section{Acknowledgements}

We thank Armando Geraldes for generously sharing data from [27].

\section{Authors' contributions}

MPR and $\mathrm{JH}$ conceived, designed, and executed the primary analyses. BH advised on project design, data preparation, and analyses. All authors interpreted results and participated in secondary analyses. MPR prepared the original draft and all authors participated in revision. All authors have read and approved the manuscript.

\section{Funding}

This work was supported by an Extreme Science and Engineering Discovery Environment (XSEDE) allocation to MPR (MCB180131). XSEDE is supported by National Science Foundation grant number ACl-1548562. MPR was funded by a University Faculty Fellowship, Monmouth University. J.H. was supported by National Science Foundation grant 1564659 (PI Arun Sethuraman) and National Institutes of Health grant S10OD020095 (PI Ronald Levy), and computation on Temple University's HPC was supported by National Science Foundation grant 1625061 and by US Army Research Laboratory contract W91 1NF-16-2-0189. The funding sources played no role in the design of the study, collection, analysis, and interpretation of data or in writing the manuscript.

\section{Availability of data and materials}

All data included in this study was published in [38] and is available from: http://wwwuser.gwdg.de/ evolbio/evolgen/wildmouse/vcf/AllMouse.vcf_90_ recalibrated_snps_raw_indels_reheader_PopSorted.PASS.vcf.gz. Input files, output files, and commands for the IMa3 analysis of the random sample of 200 loci and for the re-analysis of the nuclear loci from Geraldes et al. [27] have been deposited in Dryad (accessible under: doi:https://doi. org/10.5061/dryad.qrfj6q5cp).

\section{Ethics approval and consent to participate}

Not applicable.

Consent for publication

All authors have agreed to publication.

Competing interests

The authors declare no competing interests. 


\section{Author details}

${ }^{1}$ Department of Biology, Monmouth University, West Long Branch, NJ, USA. ${ }^{2}$ Department of Evolutionary Genetics, Max-Planck-Institute for Evolutionary Biology, Plön, Germany. ${ }^{3}$ Department of Biology, Center for Computational Genetics and Genomics, Temple University, Philadelphia, PA, USA

Received: 2 March 2020 Accepted: 27 July 2020

Published online: 15 September 2020

\section{References}

1. Morse HC. The laboratory mouse-A historical perspective. In: Foster HL, Small JD, Fox JG, editors. The mouse in biomedical research. New York: Academic Press; 1981. p. 1-16.

2. Morse HC. Building a better mouse: One hundred years of genetics and biology. In: Fox JG, Barthold SW, Davisson MT, Newcomer CE, Quimby FW, Smith SL, editors. The mouse in biomedical research. 2nd ed. San Diego: Academic Press; 2007. p. 1-11.

3. Phifer-Rixey M, Nachman MW. Insights into mammalian biology from the wild house mouse Mus musculus. eLife. 2015;4:e05959.

4. Ardlie KG, Silver LM. Low frequency of mouse t haplotypes in wild populations is not explained by modifiers of meiotic drive. Genetics. 1996; 144:1787-97.

5. Song Y, Endepols S, Klemann N, Richter D, Matuschka F-R, Shih C-H, et al. Adaptive introgression of anticoagulant rodent poison resistance by hybridization between old world mice. Curr Biol. 2011;21(15):1296-301.

6. Dod B, Jermlin LS. Counterselection on sex chromosomes in the Mus musculus European hybrid zone. J Evol Biol. 1993;6:529-46.

7. Forejt J, Iványi P. Genetic studies on male sterility of hybrids between laboratory and wild mice (Mus musculus L.). Genet Res. 1974;24(2):189-206.

8. Janoušek V, Wang L, Luzynski K, Dufková P, Vyskočilová MM, Nachman MW, et al. Genome-wide architecture of reproductive isolation in a naturally occurring hybrid zone between Mus musculus musculus and M. m. domesticus. Mol Ecol. 2012:21(12):3032-47.

9. Larson EL, Vanderpool D, Sarver BAJ, Callahan C, Keeble S, Provencio LP, et al. The evolution of polymorphic hybrid incompatibilities in house mice. Genetics. 2018;209(3):845-59.

10. Mihola O, Trachtulec Z, Vlcek C, Schimenti JC, Forejt J. A mouse speciation gene encodes a meiotic histone H3 methyltransferase. Science. 2009; 323(5912):373-5.

11. Phifer-Rixey M, Bomhoff M, Nachman MW. Genome-wide patterns of differentiation among house mouse subspecies. Genetics. 2014;198(1):28397.

12. Turner LM, Harr B. Genome-wide mapping in a house mouse hybrid zone reveals hybrid sterility loci and Dobzhansky-Muller interactions. eLife. 2014;3: e02504.

13. White MA, Steffy B, Wiltshire T, Payseur BA. Genetic dissection of a key reproductive barrier between nascent species of house mice. Genetics. 2011;189(1):289-304

14. Vanlerberghe F, Dod B, Boursot P, Bellis M, Bonhomme F. Absence of Ychromosome introgression across the hybrid zone between Mus musculus domesticus and Mus musculus musculus. Genet Res. 1986;48:191-7.

15. Parmenter MD, Gray MM, Hogan CA, Ford IN, Broman KW, Vinyard CJ, et al. Genetics of skeletal evolution in unusually large mice from Gough Island. Genetics. 2016:204(4):1559-72.

16. Phifer-Rixey M, Bi K, Ferris KG, Sheehan MJ, Lin D, Mack KL, et al. The genomic basis of environmental adaptation in house mice. PLoS Genet. 2018;14(9):e1007672.

17. Didion JP, de Villena FP-M. Deconstructing Mus gemischus: advances in understanding ancestry, structure, and variation in the genome of the laboratory mouse. Mamm Genome. 2013;24(0):1-20.

18. Britton-Davidian J, Fel-Clair F, Lopez J, et al. Postzygotic isolation between the two European subspecies of the house mouse: estimates from fertility patterns in wild and laboratory-bred hybrids. Biol J Linn Soc. 2005;84:379-93.

19. White MA, Stubbings M, Dumont BL, Payseur BA. Genetics and evolution of hybrid male sterility in house mice. Genetics. 2012;191(3):917-34.

20. Baird SJE, Macholán M. What can the Mus musculus musculus/M. m. domesticus hybrid zone tell us about speciation? In: Macholán M, Baird SJE, Munclinger P, Piálek J. Evolution of the house mouse. Cambridge: Cambridge University Press; 2012. p. 334-372.
21. Orth A, Adama T, Din W, Bonhomme P. Natural hybridization between two subspecies of the house mouse, Mus musculus domesticus and Mus musculus castaneus, near Lake Casitas, California. Genome. 1998;41(1): 104-10.

22. Jing M, Yu H-T, Bi X, Lai Y-C, Jiang W, Huang L. Phylogeography of Chinese house mice (Mus musculus musculus/castaneus): distribution, routes of colonization and geographic regions of hybridization. Mol Ecol. 2014;23(17): 4387-405.

23. Yonekawa H, Moriwaki K, Gotoh O, Miyashita N, Matsushima Y, Shi LM, et al Hybrid origin of Japanese mice "Mus musculus molossinus": evidence from restriction analysis of mitochondrial DNA. Mol Biol Evol. 1988;5(1):63-78.

24. Boursot P, Auffray JC, Britton-Davidian J, Bonhomme F. The evolution of house mice. Annu Rev Ecol Syst. 1993:24:119-52.

25. Suzuki H, Nunome M, Kinoshita G, Aplin KP, Vogel P, Kryukov AP, et al. Evolutionary and dispersal history of Eurasian house mice Mus musculus clarified by more extensive geographic sampling of mitochondrial DNA. Heredity (Edinb). 2013;111(5):375-90.

26. Duvaux L, Belkhir K, Boulesteix M, Boursot P. Isolation and gene flow: inferring the speciation history of European house mice. Mol Ecol. 2011; 20(24):5248-64.

27. Geraldes A, Basset P, Gibson B, Smith KL, Harr B, Yu H-T, et al. Inferring the history of speciation in house mice from autosomal, $X$-linked, Y-linked and mitochondrial genes. Mol Ecol. 2008;17(24):5349-63.

28. Geraldes A, Basset P, Smith KL, Nachman MW. Higher differentiation among subspecies of the house mouse (Mus musculus) in genomic regions with low recombination. Mol Ecol. 2011;20(22):4722-36.

29. Halligan DL, Oliver F, Eyre-Walker A, Harr B, Keightley PD. Evidence for pervasive adaptive protein evolution in wild mice. PLOS Genetics. 2010;6: e1000825.

30. Salcedo T, Geraldes A, Nachman MW. Nucleotide variation in wild and inbred mice. Genetics. 2007;177(4):2277-91.

31. Hey J, Nielsen R. Multilocus methods for estimating population sizes, migration rates and divergence time, with applications to the divergence of Drosophila pseudoobscura and D. persimilis. Genetics. 2004;167:747-60.

32. Hey J, Nielsen R. Integration within the Felsenstein equation for improved Markov chain Monte Carlo methods in population genetics. PNAS. 2007; 104(8):2785-90.

33. Nielsen $\mathrm{R}$, Wakeley J. Distinguishing migration from isolation: a Markov chain Monte Carlo approach. Genetics. 2001;158(2):885-96.

34. Rubinoff D, Holland BS. Between two extremes: mitochondrial DNA is neither the panacea nor the nemesis of phylogenetic and taxonomic inference. Syst Biol. 2005;54(6):952-61.

35. Keane TM, Goodstadt L, Danecek P, White MA, Wong K, Yalcin B, et al. Mouse genomic variation and its effect on phenotypes and gene regulation. Nature. 2011;477(7364):289-94.

36. White MA, Ane C, Dewey CN, Larget BR, Payseur A. Fine-scale phylogenetic discordance across the house mouse genome. PLoS Genet. 2009;5: e1000729.

37. Hey J, Chung Y, Sethuraman A, Lachance J, Tishkoff SA, Soudsa VC, Wang Y. 2018. Phylogeny estimation by integration over isolation with migration models. Mol Biol Evol. 2018;35:2805-18.

38. Kuhner MK. Coalescent genealogy samplers: windows into population history. Trends Ecol Evol. 2009:24:86-93.

39. Harr B, Karakoc E, Neme R, Teschke M, Pfeifle C, Pezer Ž, et al. Genomic resources for wild populations of the house mouse, Mus musculus and its close relative Mus spretus. Sci Data. 2016;3 Available from: https://www.ncbi. nlm.nih.gov/pmc/articles/PMC5020872/.

40. Bronson F. The reproductive ecology of the house mouse. Q Rev Biol. 1979; 54:265-99.

41. Milholland B, Dong X, Zhang L, Hao X, Suh Y, Vijg J. Differences between germline and somatic mutation rates in humans and mice. Nat Commun. 2017a:8:15183.

42. Ness RW, Zhang Y-H, Cong L, Wang Y, Zhang J-X, Keightley PD. Nuclear gene variation in wild brown rats. G3: Genes, Genomes. Genetics. 2012;2: 1661-4.

43. Tucker PK, Sage RD, Warner J, Wilson AC, Eicher EM. Abrupt cline for sex chromosomes in a hybrid zone between two species of mice. Evolution. 1992:46:1146-63.

44. Good JM, Handel MA, Nachman MW. Asymmetry and polymorphism of hybrid male sterility during the early stages of speciation in house mice. Evolution. 2008;62:50-65. 
45. Hudson RR, Slatkin M, Maddison WP. Estimation of levels of gene flow from DNA sequence data. Genetics. 1992;132(2):583.

46. Miller SA, Dykes DD, Polesky HF. A simple salting out procedure for extracting DNA from human nucleated cells. Nucleic Acids Res. 1988;16(3): 1215.

47. Mouse Genome Sequencing Consortium. Initial sequencing and comparative analysis of the mouse genome. Nature. 2002;420(6915):520-62.

48. Li H, Durbin R. Fast and accurate short read alignment with burrowswheeler transform. Bioinformatics. 2009;25(14):1754-60.

49. McKenna A, Hanna M, Banks E, Sivachenko A, Cibulskis K, Kernytsky A, et al. The genome analysis toolkit: a MapReduce framework for analyzing nextgeneration DNA sequencing data. Genome Res. 2010;20(9):1297-303.

50. Hudson RR, Kaplan NL. (722 co-authors). Statistical properties of the number of recombination events in the history of a sample of DNA sequences. Genetics. 1985:111:147-64.

51. Hey J, Wang K. The effect of undetected recombination on genealogy sampling and inference under an isolation-with-migration model. Mol Ecol Resour. 2019;19(6):1593-609.

52. Hey J. The divergence of chimpanzee species and subspecies as revealed in multipopulation isolation-with-migration analyses. Mol Biol Evol. 2010;27: 921-33.

53. Webb A, Knoblauch J, Sabankar N, Kallur AS, Hey J, Sethuraman A. The popgen pipeline platform: a software platform for facilitating population genomic analyses. bioRxiv. 2019:785774.

54. Cox A, Ackert-Bicknell CL, Dumont BL, Ding Y, Bell JT, Brockmann GA, et al. A new standard genetic map for the laboratory mouse. Genetics. 2009;182: 1335-44.

55. Geyer CJ. Markov chain Monte Carlo maximum likelihood. In: Computing Science and Statistics, Proceedings of the 23rd Symposium on the Interface; 1991. p. 156-63.

\section{Publisher's Note}

Springer Nature remains neutral with regard to jurisdictional claims in published maps and institutional affiliations.

Ready to submit your research? Choose BMC and benefit from:

- fast, convenient online submission

- thorough peer review by experienced researchers in your field

- rapid publication on acceptance

- support for research data, including large and complex data types

- gold Open Access which fosters wider collaboration and increased citations

- maximum visibility for your research: over $100 \mathrm{M}$ website views per year

At $\mathrm{BMC}$, research is always in progress.

Learn more biomedcentral.com/submissions 